\title{
Curvature flow in hyperbolic spaces
}

\author{
By Ben Andrews at Beijing/Canberra and Xuzhong Chen at Shanghai
}

\begin{abstract}
We study the evolution of compact convex hypersurfaces in hyperbolic space $\mathbb{H}^{n+1}$, with normal speed governed by the curvature. We concentrate mostly on the case of surfaces, and show that under a large class of natural flows, any compact initial surface with Gauss curvature greater than 1 produces a solution which converges to a point in finite time, and becomes spherical as the final time is approached. We also consider the higher-dimensional case, and show that under the mean curvature flow a similar result holds if the initial hypersurface is compact with positive Ricci curvature.
\end{abstract}

\section{Introduction}

In this paper, we consider compact hypersurfaces $M_{t}=X_{t}(M)$ in hyperbolic space $\mathbb{H}^{n+1}$ that contract with normal velocity equal to $F$, according to the evolution equation

$$
\frac{\partial}{\partial t} X=-F v
$$

where $F$ is a function of the principal curvatures of the surface, and $v$ is the outer unit normal of $M_{t}$. There are many papers which consider the evolution of hypersurfaces under flows of this kind, beginning with the work of Huisken [21] on mean curvature flow, and including flow by powers of Gauss curvature [15, 29], scalar curvature [16], and large classes of other examples $[2,7]$. Several previous papers have considered such flows in the special case of surfaces in three-dimensional space [4, 5, 8, 25-28], where the low-dimensional setting allows a more complete understanding of the equation for the evolution of the second fundamental form, and where there is also a more general regularity theory available [6]. In particular, in Euclidean space [4], compact convex surfaces moving by their Gauss curvature become spherical as they shrink to points, and the same is true if the speed of motion is an arbitrary monotone increasing homogeneous degree one function of the principal curvatures [8]. The authors [10] recently proved results of this kind if the speed is a power of the Gauss curvature.

The first author was partly supported by Discovery Projects grant DP1200097 of the Australian Research Council. The second author was partly supported by National Natural Science Foundation of China under grants 11271132, 11071212 and 11131007. The authors are grateful for the hospitality of the Mathematical Sciences Center of Tsinghua University where the research was carried out. 
For hypersurfaces in non-Euclidean background spaces, the understanding of the behaviour of these flows is less complete (see [5, 23, 24]). The first author [3] found a flow which takes any compact hypersurface with principal curvatures greater that $\sqrt{c}$ in a Riemannian background space with sectional curvatures at least $-c$ (with $c \geq 0$ ), and deforms it to a point with spherical limiting shape. However, this flow is rather special and the behaviour of flows such as mean curvature flow, Gauss curvature flow, and other examples are not well understood in this setting.

In this paper, we consider the corresponding questions for surfaces in hyperbolic spaces. The negative curvature of the background space produces terms which prevent the same estimates from being applied in the hyperbolic setting, and it is necessary to find different improving quantities in order to control the geometry of the evolving surfaces. We concentrate mostly on the case of surfaces, for which we study several classes of examples: If $F$ is equal to the mean curvature $H$, so that the surfaces move by the mean curvature flow, previous results [14] apply for principal curvatures $\kappa_{i}$ bounded below by 1 (one says that the surface is horospherically convex). Huisken [22] allowed the weaker condition $\kappa_{i} H>2$ for each $i$. We allow the weakest possible condition, namely positive intrinsic scalar curvature $R=2(K-1)$ : We show that the mean curvature flow preserves this condition, and evolves any compact surface with $R>0$ to a point in finite time, with spherical limiting shape. We prove a similar result for an analogue of the Gauss curvature flow, in which $F=K-1=R / 2$ : This flow contracts surfaces with positive intrinsic scalar curvature to round points. Finally, we provide a generalisation of the results of [8] to the hyperbolic setting: Let $\delta$ be the set of all smooth, homogeneous degree one symmetric functions defined on the positive cone in $\mathbb{R}^{2}$ which have strictly positive derivative in each argument on the interior of the positive cone. Such speeds include the mean curvature $H=\kappa_{1}+\kappa_{2}$, the square root of Gauss curvature $\sqrt{K}=\sqrt{\kappa_{1} \kappa_{2}}$, the power means $H_{r}=\left(\kappa_{1}^{r}+\kappa_{2}^{r}\right)^{1 / r}$ including the harmonic mean curvature $(r=-1)$, and most generally speeds of the form

$$
\tilde{F}\left(\kappa_{1}, \kappa_{2}\right)=H \varphi\left(\frac{\kappa_{2}-\kappa_{1}}{H}\right)
$$

where $\varphi$ is an arbitrary smooth positive function on $(-1,1)$ satisfying

$$
-\frac{1}{1-x}<\frac{\varphi^{\prime}(x)}{\varphi(x)}<\frac{1}{1+x} \text { for each } x \in(-1,1) .
$$

The result of [8] allowed arbitrary speeds in $\delta$, but in the hyperbolic case we instead require the form

$$
F=\left(1-\frac{1}{K}\right) \tilde{F}
$$

for arbitrary $\tilde{F} \in \mathcal{S}$. Remarkably no condition on concavity or convexity of $F$ is imposed. In the final section of the paper, we consider the higher-dimensional situation, for which we consider only the case of motion by mean curvature. The strong condition of horospherical convexity is weakened to positive intrinsic Ricci curvature.

We identify $\mathbb{H}^{n+1}$ with the future timelike unit sphere

$$
\left\{\left(x, x_{0}\right) \in \mathbb{R}^{n+1} \times \mathbb{R}: x_{0}=\sqrt{1+|x|^{2}}\right\}
$$

in Minkowski space $\mathbb{R}^{n+1,1} \simeq \mathbb{R}^{n+1} \times \mathbb{R}$, so that we can rescale embeddings into $\mathbb{H}^{n+1}$ as maps into the vector space $\mathbb{R}^{n+1,1}$. We assume that $M$ is compact and connected. Our main result for the $n=2$ case is as follows. 
Theorem 1. Let $X_{0}: M^{2} \rightarrow \mathbb{H}^{3}$ be an embedding with positive scalar curvature $R$. Assume that either $F=H, F=K-1$ or $F=(1-1 / K) \tilde{F}$ where $\tilde{F} \in 8$. Then there exists a unique solution $X: M \times[0, T) \rightarrow \mathbb{H}^{3}$ of $(1.1)$ with $X(z, 0)=X_{0}(z)$ for all $z \in M$, on a maximal time interval $[0, T)$. The surfaces $M_{t}=X(M, t)$ are strictly convex for each $t \in[0, T)$, and converge uniformly to $p \in \mathbb{H}^{3}$ as $t \rightarrow T$. The solutions are asymptotic to a shrinking sphere as $t \rightarrow T$, in the following sense: If $\mathbb{O}_{p} \in O(3,1)$ is the Lorenz boost which brings $p$ to the point $e_{0} \in \mathbb{R}^{3,1}$, then the rescalings

$$
\tilde{X}(z, t)=\frac{\mathbb{O}_{p}(X(z, t))-e_{0}}{r(t)}
$$

converge in $C^{\infty}$ to a limiting immersion $\tilde{X}_{T}$ with image equal to the unit sphere in $\mathbb{R}^{3} \subset \mathbb{R}^{3,1}$, with $\left\|\tilde{X}_{t}-\tilde{X}_{T}\right\|_{C^{k}} \leq C_{k}(T-t)^{a}$ for each $k$ for some $a>0$. Here we can choose

$$
r(t)= \begin{cases}\sqrt{4(T-t)} & \text { for } F=H, \\ \sqrt[3]{3(T-t)} & \text { for } F=K-1, \\ \sqrt{2 \tilde{F}(1,1)(T-t)} & \text { for } F=(1-1 / K) \tilde{F} .\end{cases}
$$

The key step in the proof is a pinching estimate for the principal curvatures, analogous to those used in [4] and [8]. The details of the precise pinching estimate, and the subsequent analysis, differ substantially in the three cases, so we present the proofs of each case separately.

Our result for hypersurfaces in higher-dimensional hyperbolic spaces is as follows:

Theorem 2. For any embedding $X_{0}: M^{n} \rightarrow \mathbb{H}^{n+1}$ with positive Ricci curvature, there exists a smooth solution of the mean curvature flow (equation (1.1) with $F=H$ ) on a maximal time interval $[0, T)$. The hypersurfaces $M_{t}=X_{t}(M)$ have positive Ricci curvature for each $t \in(0, T)$, and are asymptotic to a shrinking sphere as $t \rightarrow T$, in the following sense: If $\mathbb{O}_{p} \in O(n+1,1)$ is the Lorenz boost which brings $p$ to the point $e_{0} \in \mathbb{R}^{n+1,1}$, then the rescaled immersions

$$
\tilde{X}(z, t)=\frac{\mathbb{O}_{p}(X(z, t))-e_{0}}{\sqrt{2 n(T-t)}}
$$

converge in $C^{\infty}$ to a limiting immersion $\tilde{X}_{T}$ with image equal to the unit sphere in the vector space $\mathbb{R}^{n+1} \subset \mathbb{R}^{n+1,1}$, with $\left\|\tilde{X}_{t}-\tilde{X}_{T}\right\|_{C^{k}} \leq C_{k}(T-t)^{a}$ for each $k$ for some a $>0$.

We remark that restricting to hypersurfaces in $\mathbb{H}^{n+1}$ does not exclude consideration of surfaces in other hyperbolic manifolds: The assumption of positive Ricci curvature guarantees that $M$ has compact universal cover, so any immersion of $M$ into a hyperbolic manifold lifts to an immersion of the universal cover into $\mathbb{H}^{n+1}$, and the evolution equation (1.1) respects this lifting. Thus our results also hold in this more general setting.

\section{Notation and preliminary results}

Throughout the paper we adopt the Einstein summation convention of summing over repeated indices from 1 to $n$ unless otherwise specified.

We denote by $u \cdot v$ the Minkowski inner product of two vectors in $\mathbb{R}^{n+1,1}$, defined by $e_{0} \cdot e_{0}=-1$ and $e_{i} \cdot e_{i}=1$ for $i=1, \ldots, n+1$, with $e_{i} \cdot e_{j}$ for $i \neq j$. We consider 
immersions $X: M \rightarrow \mathbb{H}^{n+1} \subset \mathbb{R}^{n+1,1}$ in which the induced inner product on $T M$ defined by $g(u, v)=u X \cdot v X$ has positive intrinsic Ricci curvature (here $u X$ denotes the derivative of $X$ in direction $u$ ). At each point we have a normal line within $T_{X(x)} \mathbb{H}^{n+1}$ defined by

$$
N_{x} M=\left\{\mathbf{n} \in \mathbb{R}^{n+1,1}: \mathbf{n} \cdot u X=0 \text { for all } u \in T_{x} M, \mathbf{n} \cdot X=0\right\} .
$$

For each unit vector $v \in N_{x} M$ we have a second fundamental form $h_{v}: T_{x} M \times T_{x} M \rightarrow \mathbb{R}$ defined by $h_{v}(u, v)=-u v X \cdot v$. The principal curvatures $\kappa_{i}$ are the eigenvalues of $h_{v}$ with respect to the inner product $g$, and the principal directions are the corresponding eigenvectors; the mean curvature is $H$ is the sum of the principal curvatures, and we denote by $|A|^{2}$ the sum of the shares of the principal curvatures. The scalar curvature is given by the Gauss equation as $R=H^{2}-|A|^{2}-n(n-1)$, so the positive Ricci curvature assumption implies that $|H|>n$ everywhere. We can therefore choose the unit normal so that $H$ is positive. Henceforward we make this choice of $v$, but omit the subscript $v$ and refer to the second fundamental form simply by $h$. The Ricci curvature in the direction of the $i$ th principal direction is given by $\kappa_{i}\left(H-\kappa_{i}\right)-(n-1)$, so each $\kappa_{i}$ is positive and the hypersurface is locally convex. Given a basis $\left\{\partial_{i}\right\}$ for $T_{x} M$ we define the components of $g$ and $h$ in this basis by $g_{i j}=g\left(\partial_{i}, \partial_{j}\right)$ and $h_{i j}=h\left(\partial_{i}, \partial_{j}\right)$. The cotangent space $T^{*} M$ then has dual basis $\left\{d x^{i}\right\}$ defined by $d x^{i}\left(\partial_{j}\right)=\delta_{j}^{i}$, and the dual metric on $T^{*} M$ then has components $g^{i j}$ given by the inverse of the matrix $g_{i j}$.

The Weingarten relation provides a formula for the derivative of the unit normal vector $v$ :

$$
u v \cdot v X=h(u, v),
$$

or equivalently, with respect to a basis,

$$
\partial_{i} v=h_{i k} g^{k l} \partial_{l} X=h_{i}^{l} \partial_{l} X .
$$

The second derivatives of the immersion $X$ can be decomposed as follows:

$$
u v X=-v h(u, v)+\left(\nabla_{u} v\right) X-X g(u, v)
$$

where $\nabla$ is the Levi-Civita connection of the metric $g$. The Codazzi equation then gives

$$
\nabla_{u} h(v, w)=\nabla_{v} h(u, w)
$$

for all $u, v, w \in T_{x} M$. Combining the Gauss and Codazzi equations gives the following generalisation of Simons' identity (see for example [3]):

$$
\nabla_{(i} \nabla_{j} h_{k l}=\nabla_{(k} \nabla_{l)} h_{i j}+h_{i j} h_{k}^{p} h_{p l}-h_{k l} h_{i}^{p} h_{p j}-g_{i j} h_{k l}+g_{k l} h_{i j}
$$

where the brackets denote symmetrisation.

Also note that at a given point $p \in M$ we can always choose coordinates so that

$$
g_{i j}=\delta_{i j}, \quad \nabla_{\frac{\partial}{\partial x_{i}}} \frac{\partial}{\partial x_{j}}=0, \quad h_{j}^{i}=\operatorname{diag}\left(\kappa_{1}, \ldots, \kappa_{n}\right) .
$$

The normal velocity $F$ can be considered as a function of $\left(\kappa_{1}, \kappa_{2}\right)$ or $\left(h_{i j}, g_{i j}\right)$. We set

$$
\dot{F}^{i j}=\frac{\partial F}{\partial h_{i j}}, \quad \ddot{F}^{i j, k l}=\frac{\partial^{2} F}{\partial h_{i j} \partial h_{k l}} .
$$

Note that in an orthonormal frame with $h_{i j}=\operatorname{diag}\left(\kappa_{1}, \ldots, \kappa_{n}\right)$ we also have

$$
\dot{F}^{i j}=\operatorname{diag}\left(\dot{F}^{1}, \ldots, \dot{F}^{n}\right)
$$

where $\dot{F}^{i}=\frac{\partial F}{\partial \kappa_{i}}$. 
Under the evolution equation (1.1) we have the following evolution equations (see [3]):

$$
\begin{aligned}
& \frac{\partial}{\partial t} g_{i j}=-2 F h_{i j} \\
& \frac{\partial}{\partial t} F= \dot{F}^{i j} \nabla_{i} \nabla_{j} F+F \dot{F}^{i j} h_{i p} h_{j}^{p}-F \dot{F}^{i j} g_{i j} \\
& \frac{\partial}{\partial t} h_{i j}= \dot{F}^{k l} \nabla_{k} \nabla_{l} h_{i j}+\ddot{F}^{k l, m n} \nabla_{i} h_{k l} \nabla_{j} h_{m n}-\dot{F}^{k l} h_{k l} h_{i}^{p} h_{p j}+\dot{F}^{k l} h_{i j} h_{k}^{p} h_{p l} \\
&-\dot{F}^{k l} h_{k l} g_{i j}+\dot{F}^{k l} g_{k l} h_{i j}-F h_{i}^{k} h_{k j}-F g_{i j} \\
& \frac{\partial}{\partial t} G=\dot{F}^{i j} \nabla_{i} \nabla_{j} G+\left(\dot{G}^{i j} \ddot{F}^{k l, m n}-\dot{F}^{i j} \ddot{G}^{k l, m n}\right) \nabla_{i} h_{k l} \nabla_{j} h_{m n} \\
&-\dot{G}^{i j} \dot{F}^{k l} h_{k l} h_{i}^{p} h_{p j}+\dot{G}^{i j} \dot{F}^{k l} h_{i j} h_{k}^{p} h_{p l}-\dot{G}^{i j} \dot{F}^{k l} g_{i j} h_{k l} \\
&+\dot{G}^{i j} \dot{F}^{k l} g_{k l} h_{i j}+F \dot{G}^{i j} h_{i}^{k} h_{k j}-F \dot{G}^{i j} g_{i j}
\end{aligned}
$$

where $G=G\left(h_{j}^{i}\right)$ is a symmetric function of the principal curvatures. In the special case $n=2, F=K-1$ this becomes

$$
\begin{aligned}
\frac{\partial}{\partial t} g_{i j} & =-2(K-1) h_{i j}, \\
\frac{\partial}{\partial t} H & =\dot{K}^{i j} \nabla_{i} \nabla_{j} H+g^{i j} \ddot{K}^{i j, k l} \nabla_{i} h_{k l} \nabla_{j} h_{m n}+2(K-1)^{2}, \\
\frac{\partial}{\partial t} K & =\dot{K}^{i j} \nabla_{i} \nabla_{j} K+(K-1)^{2} H .
\end{aligned}
$$

Finally, for $F=H$, we have the following:

$$
\begin{aligned}
\frac{\partial}{\partial t} g_{i j} & =-2 H h_{i j}, \\
\frac{\partial}{\partial t} h_{i j} & =\Delta h_{i j}-2 H\left(h_{i k} h_{j}^{k}+g_{i j}\right)+\left(|A|^{2}+n\right) h_{i j}, \\
\frac{\partial}{\partial t} H & =\Delta H+\left(|A|^{2}-n\right) H .
\end{aligned}
$$

If $F$ is a smooth function of the principal curvatures defined on some open subset $A$ of $\mathbb{R}^{n}$, which is strictly increasing in each argument, and $X_{0}$ is a smooth immersion of a compact $n$-manifold as a hypersurface in $\mathbb{H}^{n+1}$ such that the principal curvatures at each point lie in $A$, then there exists a unique smooth solution for a short time interval (see for example the treatment given in [13]). We define $T$ to be the maximal interval of existence of a solution. In the remainder of the paper our argument will show that for the particular classes of speed $F$ we consider, the evolving hypersurfaces contract to points and become spherical in shape at the end of the maximal interval of existence.

We conclude this section by observing that the maximal time of existence is necessarily finite for a very large class of flows:

Proposition 1. Suppose the normal velocity $F$ is defined and positive on a domain $A$ which contains $(c, \ldots, c)$ for $c>1$, and is nondecreasing (in the sense that if $\left(a_{1}, \ldots, a_{n}\right)$ and $\left(b_{1}, \ldots, b_{n}\right)$ are in $A$ with $b_{i} \geq a_{i}$ for all $i$, then $\left.F\left(b_{1}, \ldots, b_{n}\right) \geq F\left(a_{1}, \ldots, a_{n}\right)\right)$. Then the maximal time of existence for any compact solution of (1.1) is finite. 
Proof. Let $d$ be the hyperbolic distance from any fixed point in $\mathbb{H}^{3}$. Then $d$ is smooth where it is nonzero, and we have the following evolution equation:

$$
\frac{\partial d}{\partial t}=D d(-F v)
$$

At a point where the spatial maximum of $d$ is attained, we have

$$
0=\nabla_{i} d=\operatorname{Dd}\left(\partial_{i}\right)
$$

so that $v$ points in the radial direction, and

$$
0 \geq \nabla_{j} \nabla_{i} d=D^{2} d\left(\partial_{i}, \partial_{j}\right)-D d\left(h_{i j} v\right) .
$$

But since $v$ is radial, we have

$$
D^{2} d\left(\partial_{i}, \partial_{j}\right)=\operatorname{coth} d g_{i j}
$$

so the second derivative condition becomes

$$
\kappa_{i} \geq \operatorname{coth} d
$$

for all $i$. Since $F$ is an increasing function of the principal curvatures, this implies that

$$
F \geq F(\operatorname{coth} d, \ldots, \operatorname{coth} d)>0 .
$$

The maximum principle (in the form given in [19, Lemma 3.5]) implies that the maximum of $d$ is non-increasing, so we have $d \leq d_{0}=\sup _{x \in M} d(X(x, 0))$. The monotonicity of $F$ and the fact that coth is a decreasing function then gives

$$
F \geq F_{0}=F\left(\operatorname{coth} d_{0}, \ldots, \operatorname{coth} d_{0}\right),
$$

and so

$$
\frac{\partial}{\partial t} d \leq-F_{0}
$$

so that

$$
\sup _{x \in M} d(X(x, t)) \leq d_{0}-F_{0} t .
$$

Since $d$ is manifestly non-negative, we conclude that $T \leq d_{0} / F_{0}$.

\section{Scalar curvature flow}

In this section, we will study the $K-1$ flow for $n=2$ :

$$
\frac{\partial}{\partial t} X=-(K-1) v
$$

where $K$ is Gauss curvature and $v$ is the outer normal vector of $M_{t}$. In this case, the crucial estimate is a bound on the difference between the principal curvatures, which follows a similar argument to that in [4]. 
3.1. Pinching estimate. We first prove that positive scalar curvature of the initial surface is preserved by equation (3.1):

Proposition 2. If $K(x, 0)>1$ for all $x \in M$, then $K(x, t)>1$ for all $x \in M$ and all $t \in[0, T)$ under (3.1). As a consequence, the surfaces $M_{t}$ are strictly convex for all $t \in[0, T)$.

Proof. This follows from the evolution equation (2.11) for Gauss curvature $K$, and maximum principle.

Next we prove the bound on the difference between the principal curvatures:

Theorem 3. Let $\left\{M_{t}=X(M, t)\right\}_{0 \leq t \leq T}$ be a smooth connected solution of the flow equation (3.1) with $K>1$. Then

$$
\sup _{p \in M}\left|\kappa_{1}(p, t)-\kappa_{2}(p, t)\right| \leq \sup _{p \in M}\left|\kappa_{1}(p, 0)-\kappa_{2}(p, 0)\right| .
$$

Proof. We will apply the maximum principle to the quantity

$$
Q=H^{2}-4 K=\left(\kappa_{1}-\kappa_{2}\right)^{2} .
$$

We first compute an evolution equation for $Q$ :

$$
\begin{aligned}
\frac{\partial Q}{\partial t}= & 2 H \frac{\partial H}{\partial t}-4 K \frac{\partial K}{\partial t} \\
= & 2 H\left(\dot{K}^{i j} \nabla_{i} \nabla_{j} H+g^{i j} \ddot{K}^{i j, k l} \nabla_{i} h_{k l} \nabla_{j} h_{m n}+2(K-1)^{2}\right) \\
& \quad-4\left(\dot{K}^{i j} \nabla_{i} \nabla_{j} K+(K-1)^{2} H\right) \\
= & \dot{K}^{i j} \nabla_{i} \nabla_{j} Q-2 \dot{K}^{i j} \nabla_{i} H \nabla_{j} H+2 H g^{i j} \ddot{K}\left(\nabla_{i} h, \nabla_{j} h\right) .
\end{aligned}
$$

Suppose $p$ is a point in $M$ where a maximum of $Q$ is attained at time $t \in[0, T)$, choose local coordinates for $M$ near $p$ such that $g_{i j}=\delta_{i j}$ and $h_{i j}$ is diagonal. At this point the leading term on the right-hand side of the above evolution equation is non-positive. We now estimate the remaining terms. Using the fact that $\nabla Q=0$ at $p$,

$$
\begin{aligned}
0 & =\nabla_{1} Q \\
& =2 H \nabla_{1} H-4 \nabla_{1} K \\
& =2\left(\kappa_{1}+\kappa_{2}\right)\left(\nabla_{1} h_{11}+\nabla_{2} h_{22}\right)-4 \kappa_{1} \nabla_{1} h_{22}-4 \kappa_{2} \nabla_{1} h_{11} \\
& =2\left(\kappa_{1}-\kappa_{2}\right)\left(\nabla_{1} h_{11}-\nabla_{1} h_{22}\right) .
\end{aligned}
$$

If $\kappa_{1}=\kappa_{2}$, then $Q=0$ and we have nothing to prove. So we can assume that $\nabla_{1} h_{11}=\nabla_{1} h_{22}$ at the point $p$. Similarly, we have $\nabla_{2} h_{11}=\nabla_{2} h_{22}$. Now we compute

$$
\begin{aligned}
\ddot{K}\left(\nabla_{1} h, \nabla_{1} h\right) & =2 \nabla_{1} h_{11} \nabla_{1} h_{22}-2\left(\nabla_{1} h_{12}\right)^{2} \\
& =2\left(\nabla_{1} h_{11}\right)^{2}-2\left(\nabla_{2} h_{11}\right)^{2} \\
& =2\left(\nabla_{1} h_{11}\right)^{2}-2\left(\nabla_{2} h_{22}\right)^{2}
\end{aligned}
$$

by using $\nabla Q=0$ condition and the Codazzi identity. Similarly,

$$
\ddot{K}\left(\nabla_{2} h, \nabla_{2} h\right)=2\left(\nabla_{2} h_{22}\right)^{2}-2\left(\nabla_{1} h_{11}\right)^{2},
$$


therefore at the point $p$, we have

$$
g^{i j} \ddot{K}\left(\nabla_{i} h, \nabla_{j} h\right)=\ddot{K}\left(\nabla_{1} h, \nabla_{1} h\right)+\ddot{K}\left(\nabla_{2} h, \nabla_{2} h\right) .
$$

Thus the last term on the right-hand side of the evolution equation for $Q$ vanishes and the second term is manifestly non-positive. The maximum principle applies to show that the supremum of $Q$ over $M$ is non-increasing function of time.

Corollary 4. Under the conditions of Theorem 3 there exists a constant $C_{1}$ such that

$$
0<\frac{1}{C_{1}} \leq \frac{\kappa_{2}}{\kappa_{1}} \leq C_{1} .
$$

Proof. Theorem 3 and the inequality $K>1$ imply that

$$
\frac{\kappa_{1}}{\kappa_{2}}+\frac{\kappa_{2}}{\kappa_{1}}-2=\frac{\left(\kappa_{1}-\kappa_{2}\right)^{2}}{\kappa_{1} \kappa_{2}} \leq\left(\kappa_{1}-\kappa_{2}\right)^{2} \leq C_{0}
$$

where $C_{0} \equiv \sup _{p \in M}\left(\kappa_{1}(p, 0)-\kappa_{2}(p, 0)\right)^{2}$.

Corollary 5. Under the conditions of Theorem 3 there exists a constant $C_{2}$ such that

$$
\kappa_{i} \geq C_{2} \quad \text { for } i=1,2 .
$$

Proof. This is a direct consequence of Corollary 4 and the inequality $K>1$.

3.2. Convergence. In this subsection, we discuss the convergence of the solution to a point and of the rescaled immersions to the sphere. The argument differs from that in previous work $[3,27]$ in only a few points. As in other flows with speed growing super linearly in the curvature, the main difficulty is in the non-uniform parabolicity of the flow. This difficulty can be overcome using either the methods of $[1,28,30]$ for which the key step is to apply estimates for porous medium equations, or the methods of [12] which use geometric estimates to show the surface becomes close to a sphere near the final time, and then a barrier argument to force the speed to become positive so that the flow becomes uniformly parabolic. We sketch here the former approach.

We first observe that the Gauss curvature must become unbounded as the final time is approached: If not, then since we have a positive lower bound on $K-1$ by Proposition 2, and a bound on ratios of principal curvatures by Corollary 4, all principal curvatures are bounded above and below by positive constants. In particular, the coefficients $\dot{F}^{i j}$ have eigenvalues bounded above and below by positive constants. The results of [6] (applied in a local graph parametrisation, for example) apply to give $C^{0, \alpha}$ bounds on the second fundamental form, and higher derivative estimates follow by standard Schauder estimates. It follows that the solution can be extended further, following an argument similar to that in [21].

We therefore consider a sequence of times $t_{k}$ approaching $T$, for which a new maximum of the Gauss curvature is attained, so that

$$
\lambda_{k}^{2}=\sup _{x \in M, 0 \leq t \leq t_{k}} K(x, t)=K\left(x_{k}, t_{k}\right)
$$

for some $x_{k} \in M$ for each $k$. Now let $\mathbb{O}_{k} \in O(3,1)$ be such that $\mathbb{O}_{k}\left(X\left(x_{k}, t_{k}\right)=e_{0}\right.$ and $v\left(x_{k}, t_{k}\right)=e_{3}$, and define

$$
X_{k}(x, t)=\lambda_{k}\left(\mathbb{O}_{k} X\left(x, t_{k}+\lambda_{k}^{-3} t\right)-e_{0}\right) .
$$


Then $X_{k}: M \times\left[-\lambda_{k}^{3} t_{k}, \lambda_{k}^{3}\left(T-t_{k}\right)\right) \rightarrow \lambda_{k}\left(\mathbb{H}^{3}-e_{0}\right)$ is a solution of the equation

$$
\frac{\partial X_{k}}{\partial t}=-\left(K_{k}-\lambda_{k}^{-2}\right) v_{k}
$$

where $K_{k}$ is the Gauss curvature of $X_{k}$ and $v_{k}$ is the unit normal (within $\lambda_{k}\left(\mathbb{H}^{3}-e_{0}\right)$ ). Furthermore, we have

$$
\sup _{x \in M, t \leq 0} K_{k}(x, t)=K_{k}\left(x_{k}, 0\right)=1 \quad \text { for each } x,
$$

$\left.v_{k}(M, 0)\right)=e_{3}$ and $X_{k}\left(x_{k}, 0\right)=0$.

The result of Corollary 4 implies that ratios of principal curvatures are bounded at each point for $X_{k}$, and hence that all principal curvatures are bounded since $K \leq 1$.

Under equation (3.5) the Gauss curvature evolves as follows:

$$
\frac{\partial}{\partial t} K=\dot{K}^{i j} \nabla_{i} \nabla_{j} K+\left(K-\lambda_{k}^{-2}\right)^{2} H .
$$

The evolving surfaces may be written locally as graphs over a totally geodesic hyperbolic 2-plane $H$ in $\mathbb{H}^{3}$ : At each point $x \in H$ let $v(x)$ be the unit normal. Then we define the map $\Psi: H \times \mathbb{R} \rightarrow \mathbb{H}^{3}$ by $\Psi(x, s)=\exp _{x}(s v(x))$, and describe $M_{t}$ locally by the embedding

$$
\tilde{X}: x \mapsto \Psi(x, u(x, t)) .
$$

The inner product induced by $\Psi$ is given by

$$
g\left(\Psi_{*}(u, a), \Psi_{*}(v, b)\right)=\cosh ^{2}\left(\lambda_{k}^{-1} s\right) \bar{g}(u, v)+a b
$$

where $\bar{g}$ is the hyperbolic metric on $H$. It follows that the tangent vectors to the embedding are given by

$$
\partial_{i} X=\partial_{i} \Psi+u_{i} \partial_{s} \Psi
$$

where $u_{i}=\partial_{i} u$, so that the induced inner product is

$$
g_{i j}=\cosh ^{2}\left(\lambda_{k}^{-1} u\right) \bar{g}_{i j}+u_{i} u_{j}
$$

and the unit normal to the surface is given by the expression

$$
v=\frac{-u_{i} \bar{g}^{i j} \partial_{j} \Psi+\cosh ^{2}\left(\lambda_{k}^{-1} u\right) \partial_{s} \Psi}{\cosh \left(\lambda_{k}^{-1} u\right) \sqrt{\cosh ^{2}\left(\lambda_{k}^{-1} u\right)+|D u|_{\bar{g}}^{2}}} .
$$

The evolution of the graphical embedding is related to $X$ by a time-dependent diffeomorphism defined by the requirement that the orthogonal projection onto $H$ remains fixed. Explicitly, this is given by

$$
\frac{\partial \tilde{X}}{\partial t}=-\left(K-\lambda_{k}^{-2}\right) v-V^{k} \partial_{k} X
$$

where

$$
V^{k}=\frac{\left(K-\lambda_{k}^{-2}\right) u_{p} \bar{g}^{p k}}{\cosh \left(\lambda_{k}^{-1} u\right) \sqrt{\cosh ^{2}\left(\lambda_{k}^{-1} u\right)+|D u|_{\bar{g}}^{2}}} .
$$


It follows that the evolution of $K$ under the graphical flow is governed by the equation

$$
\frac{\partial K}{\partial t}=\dot{K}^{i j} \nabla_{i} \nabla_{j} K+\left(K-\lambda_{k}^{-2}\right)^{2} H-V^{k} \nabla_{k} K .
$$

The Codazzi identity implies that $\nabla_{i} \dot{K}^{i j}=0$, so we also have the following divergence form:

$$
\begin{aligned}
\frac{\partial K}{\partial t} & =\nabla_{i}\left(\dot{K}^{i j} \nabla_{j} K\right)+\left(K-\lambda_{k}^{-2}\right)^{2} H-V^{k} \nabla_{k} K \\
& =\bar{\nabla}_{i}\left(\dot{K}^{i j} \bar{\nabla}_{j} K\right)+\left((\Gamma-\bar{\Gamma})_{i p}{ }^{p} \dot{K}^{p j}-V^{j}\right) \bar{\nabla}_{j} K+\left(K-\lambda_{k}^{-2}\right)^{2} H
\end{aligned}
$$

where $\Gamma_{i j}{ }^{k}$ and $\bar{\Gamma}_{i j}{ }^{k}$ are the Christoffel symbols of the Levi-Civita connections of $g$ and $\bar{g}$ on $H$, respectively. These are related by the Levi-Civita formula

$$
\Gamma_{i j}^{k}-\bar{\Gamma}_{i j}^{k}=\frac{1}{2} g^{k q}\left(\bar{\nabla}_{i} g_{j q}+\bar{\nabla}_{j} g_{i q}-\bar{\nabla}_{q} g_{i j}\right),
$$

which implies that

$$
(\Gamma-\bar{\Gamma})_{i p}{ }^{i}=\frac{1}{2} \bar{\nabla}_{p} \log \operatorname{det} g .
$$

The bound on curvature, together with barrier arguments as in [3, Lemma 5.2], gives the following: There exists an $r>0$ such that for each $k$ and each $x \in M$, the hypersurfaces can be written as hyperbolic graphs of the form (3.7) on a region

$$
\mathscr{E}_{r}=\left\{(z, t): z \in B_{r}(x),-r^{2} \leq t \leq 0\right\},
$$

with $0 \leq u(z, t) \leq r^{-1},|\bar{\nabla} u(z, t)|_{\bar{g}} \leq r^{-1}$, and $\left|\bar{\nabla}^{2} u(z, t)\right|_{\bar{g}} \leq r^{-1}$ for all $(z, t) \in \mathcal{E}_{r}$. From equation (3.15) we deduce that on each of these solutions the Gauss curvature evolves according to an equation of the form

$$
K_{t}=\bar{\nabla}_{i}\left(a^{i j} \bar{\nabla}_{j} K^{3 / 2}\right)+A^{i} \bar{\nabla}_{i} K+f
$$

where $\lambda \delta^{i j} \leq a^{i j} \leq \Lambda \delta^{i j}$ for some $0<\lambda<\Lambda$, and $A^{i}$ and $f$ are bounded. This follows since $\dot{K}^{i j} K^{-1 / 2}=K^{1 / 2}\left(h^{-1}\right)^{i j}$ has positive bounds above and below by Corollary 4 .

Theorem 1.2 of [17] can now be applied to give a Hölder continuity estimate for $K$ on the region $\mathscr{E}_{r / 2}$, with constant depending on $\int_{\mathscr{E}_{r}}\left|\bar{\nabla} K^{3 / 2}\right|^{2} d \mu(\bar{g})$. To bound the latter we observe that (since $\dot{K}^{i j}$ is comparable to $K^{1 / 2} g^{i j}$ ),

$$
\begin{aligned}
\frac{d}{d t} \int_{M} K^{5 / 2} & \leq-\frac{5}{2} \int_{M} K^{3 / 2}\left(\nabla_{i} \dot{K}^{i j} \nabla_{j} K+\left(K-\lambda_{k}^{-2}\right)^{2} H\right)+K^{5 / 2} H\left(K-\lambda_{k}^{-2}\right) d \mu \\
& \leq-C \int_{M}\left|\nabla_{i} K^{3 / 2}\right|^{2}+C,
\end{aligned}
$$

so that (since $g$ and $\bar{g}$ are comparable on $\mathscr{E}_{r}$ )

$$
\begin{aligned}
\int_{\mathscr{g}_{r}}\left|\bar{\nabla} K^{3 / 2}\right|_{\bar{g}}^{2} d \mu(\bar{g}) & \leq C \int_{M_{t}}\left|\nabla K^{3 / 2}\right|_{g}^{2} d \mu(g) \\
& \leq-C \int_{t=-r^{2}}^{t=0}\left(\frac{d}{d t} \int_{M_{t}} K^{5 / 2} d \mu(g)+C\right) d t
\end{aligned}
$$

Integrating this gives the required bound. 
This proves that $K$ is Hölder continuous on $M \times\left[-r^{2} / 2,0\right]$, with constants independent of $k$. Therefore in this region for any $\left(x_{0}, t_{0}\right)$ we have $K$ comparable to $K\left(x_{0}, t_{0}\right)$ on $B_{r}\left(x_{0}\right) \times\left[t_{0}-r^{2}, t_{0}\right]$ where $r^{2}$ is comparable to $K\left(x_{0}, t_{0}\right)$. On this set the evolution equation is uniformly parabolic, and the estimates of [6] apply to give Hölder continuity of second derivatives, with estimates depending on $K\left(x_{0}, t_{0}\right)$. Schauder estimates then give $C^{k, \alpha}$ estimates for every $k$. This proves that the hypersurfaces $M_{t}$ have all derivatives of second fundamental form bounded on regions where $K>0$.

It now follows that $X_{k}$ converges as $k \rightarrow \infty$ to a solution of Gauss curvature flow with bounded curvature in $\mathbb{R}^{3}$, which is smooth at points where $K>0$. The pinching estimate of Theorem 3 implies that $\left|\kappa_{2}-\kappa_{1}\right| \leq C \lambda_{k}^{-1} \rightarrow 0$, so the limiting hypersurface is totally umbilic and hence a sphere. The smooth (rather than subsequential) convergence follows as in [4].

\section{Mean curvature flow $(n=2)$}

In this section, we will study mean curvature flow in three-dimensional hyperbolic space:

$$
\frac{\partial}{\partial t} X=-H v
$$

where $H$ is mean curvature and $v$ is outer normal vector of $M_{t}$.

4.1. Pinching estimates. We will prove that scalar curvature of solutions to equation (4.1) remains positive if initially so, and also that the principal curvatures remain bounded in ratio. According to evolution equation (2.13), we have

$$
\frac{\partial}{\partial t} h_{i j}=\Delta h_{i j}-2 H\left(h_{i k} h_{j}^{k}+g_{i j}\right)+\left(|A|^{2}+2\right) h_{i j} .
$$

If we introduce the canonical spacetime connection (as in [11, Section 6.3] or [9, Section 2.3]) by setting $\nabla_{t} \partial_{i}=-H h_{i}^{j} \partial_{j}$, then this becomes

$$
\nabla_{t} h_{i j}=\Delta h_{i j}-2 H g_{i j}+\left(|A|^{2}+2\right) h_{i j} .
$$

We apply the vector bundle maximum principle to the evolution equation (4.2) to prove that the inequality $K>1$ is preserved by mean curvature flow.

Proposition 3. If $K>1$ at $t=0$, then $K>1$ for all $t \in[0, T)$. In particular, the surface $M_{t}$ remains strictly convex.

Proof. We will use the vector bundle maximum principle introduced in [19] (see in particular the formulation in [11, Theorem 7.15]). Let $\kappa_{1}$ and $\kappa_{2}$ be the principal curvatures of $M_{t}$. Let $Q_{i j}=\left(|A|^{2}+2\right) h_{i j}-2 H g_{i j}$, which is the reaction term of (4.2). In particular, if we work in a frame where $\left(h_{i j}\right)$ is diagonal, then so is $Q$ :

$$
h=\left[\begin{array}{cc}
\kappa_{1} & 0 \\
0 & \kappa_{2}
\end{array}\right] \Longrightarrow Q=\left[\begin{array}{cc}
\kappa_{1}^{3}+\kappa_{1} \kappa_{2}^{2}-2 \kappa_{2} & 0 \\
0 & \kappa_{2}^{3}+\kappa_{2} \kappa_{1}^{2}-2 \kappa_{1}
\end{array}\right] .
$$

Consider the following convex domain:

$$
\Omega=\left\{\left(\kappa_{1}, \kappa_{2}\right): \kappa_{1} \kappa_{2}-1 \geq 0, \kappa_{1}+\kappa_{2}>0\right\} .
$$


For the vector bundle maximum principle to apply, we need the vector field $Q$ to point into $\Omega$, which is equivalent to the derivative of the defining function $\kappa_{1} \kappa_{2}-1$ in direction $Q$ being positive. We have

$$
Q \kappa_{i}=\kappa_{i}\left(|A|^{2}+2\right)-2 H
$$

and so

$$
\begin{aligned}
Q\left(\kappa_{1} \kappa_{2}\right) & =\kappa_{1} \kappa_{2}\left(|A|^{2}+2\right)-2 H \kappa_{2}+\kappa_{1} \kappa_{2}\left(|A|^{2}+2\right)-2 H \kappa_{1} \\
& =2\left(\kappa_{1} \kappa_{2}-1\right)|A|^{2}+2|A|^{2}+4 \kappa_{1} \kappa_{2}-2 H^{2} \\
& =2\left(\kappa_{1} \kappa_{2}-2\right)|A|^{2} \\
& =0 .
\end{aligned}
$$

Therefore the maximum principle applies, and the proposition is proved.

Theorem 6. Let $\left\{M_{t}=X(M, t)\right\}_{0 \leq t<T}$ be a smooth, strictly convex solution of the flow equation (4.1) with $K>1$. Then

$$
\sup _{p \in M} \frac{\left|\kappa_{1}^{2}(p, t)-\kappa_{2}^{2}(p, t)\right|}{\kappa_{1}(p, t) \kappa_{2}(p, t)-1} \leq \sup _{p \in M} \frac{\left|\kappa_{1}^{2}(p, 0)-\kappa_{2}^{2}(p, 0)\right|}{\kappa_{1}(p, 0) \kappa_{2}(p, 0)-1} .
$$

Proof. Let

$$
C_{3}\left(M_{0}\right)=\sup _{p \in M} \frac{\left|\kappa_{1}^{2}(p, 0)-\kappa_{2}^{2}(p, 0)\right|}{\kappa_{1}(p, 0) \kappa_{2}(p, 0)-1} .
$$

We need to prove

$$
\left|\kappa_{1}^{2}-\kappa_{2}^{2}\right| \leq C_{3}\left(\kappa_{1} \kappa_{2}-1\right) .
$$

We again apply the vector bundle maximum principle. To apply this we must verify that (4.6) defines a subset of the bundle of symmetric 2-tensors which is convex in the fibre and invariant under parallel transport, and such that the vector field $Q$ defined by (4.3) points into the set. We verify this using arguments similar to [11, Section 7.5.3.1-7.5.3.2]: First we show that this defines a convex subset $\Omega$ of the vector bundle of symmetric 2-tensors: Define

$$
\Omega=\left\{h>0, h\left(e_{1}, e_{1}\right)-h\left(e_{2}, e_{2}\right) \leq C_{3}\left(\frac{\operatorname{det} h-1}{\operatorname{trace} h}\right) \text { for all }\left\{e_{1}, e_{2}\right\} \text { orthonormal }\right\} .
$$

Since $\frac{\operatorname{det} h}{\text { trace } h}$ and $-\frac{1}{\text { trace } h}$ are concave functions on the positive cone, the function

$$
h\left(e_{1}, e_{1}\right)-h\left(e_{2}, e_{2}\right)-C_{3}\left(\frac{\operatorname{det} h-1}{\operatorname{trace} h}\right)
$$

is convex for each orthonormal frame $\left\{e_{1}, e_{2}\right\}$. It follows that $\Omega$ is an intersection of convex sets, hence convex. The invariance under parallel transport is automatic from the definition. It remains to check that the vector field $Q$ points into $\Omega$. Let $h \in \partial \Omega$, so that for some frame we have

$$
h\left(e_{1}, e_{1}\right)-h\left(e_{2}, e_{2}\right)-C_{3}\left(\frac{\operatorname{det} h-1}{\operatorname{trace} h}\right) .
$$

Variation of the frame shows that $e_{1}$ and $e_{2}$ are eigenvectors of $h$, with corresponding eigenvalues $\kappa_{1}>\kappa_{2}$ satisfying $\kappa_{1}^{2}-\kappa_{2}^{2}=C_{3}\left(\kappa_{1} \kappa_{2}-1\right)$. The supporting linear function at this point is given by

$$
\ell(A)=\left[2 \kappa_{1}-C \kappa_{2}\right] A_{11}+\left[-2 \kappa_{2}-C \kappa_{1}\right] A_{22} .
$$


We have $Q_{11}=\kappa_{1}^{3}+\kappa_{2} \kappa_{2}^{2}-2 \kappa_{2}$ and $Q_{22}=\kappa_{2}^{3}+\kappa_{2} \kappa_{1}^{2}-2 \kappa_{1}$, so this becomes

$$
\begin{aligned}
\ell(Q)= & 2 \kappa_{1}^{2}\left(\kappa_{1}^{2}+\kappa_{2}^{2}\right)-4 \kappa_{1} \kappa_{2}-C \kappa_{2} \kappa_{2}\left(\kappa_{1}^{2}+\kappa_{2}^{2}\right)+2 C \kappa_{2}^{2} \\
& \quad-2 \kappa_{2}^{2}\left(\kappa_{1}^{2}+\kappa_{2}^{2}\right)+4 \kappa_{1} \kappa_{2}-C \kappa_{2} \kappa_{2}\left(\kappa_{1}^{2}+\kappa_{2}^{2}\right)+2 C \kappa_{1}^{2} \\
= & 2\left(\kappa_{1}^{2}+\kappa_{2}^{2}\right)\left(\kappa_{1}^{2}-\kappa_{2}^{2}-C\left(\kappa_{1} \kappa_{2}-1\right)\right) \\
= & 0 .
\end{aligned}
$$

The maximum principle therefore applies, and the inequality is preserved by the flow.

Corollary 7. For a smooth compact strictly convex solution of mean curvature flow with $K>1$ in $\mathbb{H}^{3}$, there exists a constant $C_{4}$ such that

$$
0<\frac{1}{C_{4}} \leq \frac{\kappa_{2}}{\kappa_{1}} \leq C_{4}
$$

Proof. Theorem 6 and $K>1$ implies that

$$
\left(\frac{\kappa_{1}}{\kappa_{2}}\right)^{2}+\left(\frac{\kappa_{2}}{\kappa_{1}}\right)^{2}-2=\frac{\left(\kappa_{1}-\kappa_{2}\right)^{2}\left(\kappa_{1}+\kappa_{2}\right)^{2}}{\left(\kappa_{1} \kappa_{2}\right)^{2}} \leq \frac{\left(\kappa_{1}-\kappa_{2}\right)^{2}\left(\kappa_{1}+\kappa_{2}\right)^{2}}{\left(\kappa_{1} \kappa_{2}-1\right)^{2}} \leq C_{3}^{2} .
$$

The corollary follows with

$$
C_{4}^{2}=1+\frac{C_{3}^{2}}{2}+C_{3} \sqrt{1+\frac{C_{3}^{2}}{4}}
$$

4.2. Convergence. The argument for convergence for the mean curvature flow is considerably simpler that for the scalar curvature flow of the previous section. We rescale in a similar way to the previous section, choosing a sequence of times $t_{k}$ on which the mean curvature reaches a mew maximum, so that

$$
\lambda_{k}=\sup _{x \in M, 0 \leq t \leq t_{k}} H(x, t)=H\left(x_{k}, t_{k}\right)
$$

for some $x_{k} \in M$ for each $k$. Now let $\mathbb{O}_{k} \in O(3,1)$ be such that $\mathbb{O}_{k}\left(X\left(x_{k}, t_{k}\right)=e_{0}\right.$ and $v\left(x_{k}, t_{k}\right)=e_{3}$, and define

$$
X_{k}(x, t)=\lambda_{k}\left(\mathbb{O}_{k} X\left(x, t_{k}+\lambda_{k}^{-2} t\right)-e_{0}\right) .
$$

Then $X_{k}: M \times\left[-\lambda_{k}^{2} t_{k}, \lambda_{k}^{2}\left(T-t_{k}\right)\right) \rightarrow \lambda_{k}\left(\mathbb{H}^{3}-e_{0}\right)$ is a solution of the mean curvature flow. Furthermore, we have

$$
\sup _{x \in M, t \leq 0} H_{k}(x, t)=H_{k}\left(x_{k}, 0\right)=1 \quad \text { for each } x,
$$

$\left.v_{k}(M, 0)\right)=e_{3}$ and $X_{k}\left(x_{k}, 0\right)=0$.

Standard estimates (see for example [18, Theorem 3.4] for the Euclidean mean curvature flow) yield bounds on all higher derivatives of second fundamental form, independent of $k$. It follows that the solutions $X_{k}$ converge to a complete strictly convex solution of mean curvature flow in Euclidean space, satisfying the pinching ratio bound of Corollary 7 . The result 
of [20] implies that the limiting solution consists of compact convex hypersurfaces, and the fact that the pinching ratio of a compact convex solution of mean curvature flow in Euclidean space is strictly decreasing unless the hypersurface is a sphere implies that the limiting is a shrinking sphere solution. The convergence result follows.

\section{5. $F$-flow}

In this section, we will study the flow in three-dimensional hyperbolic space:

$$
\frac{\partial}{\partial t} X=-F v
$$

where $F=\left(1-\frac{1}{K}\right) \tilde{F}$ and $v$ is the outer normal vector of $M_{t}$.

5.1. Pinching estimates. The crucial estimate in this case is a bound on the ratio of principal curvatures.

Theorem 8. Let $\left\{M_{t}=X(M, t)_{0 \leq t<T}\right\}$ be a smooth, solution of the flow equation (5.1) with $K>1$. Then

$$
\sup _{M} \frac{\left(\kappa_{1}(x, t)-\kappa_{2}(x, t)\right)^{2}}{\left(\kappa_{1}(x, t)+\kappa_{2}(x, t)\right)^{2}} \leq \sup _{M} \frac{\left(\kappa_{1}(x, 0)-\kappa_{2}(x, 0)\right)^{2}}{\left(\kappa_{1}(x, 0)+\kappa_{2}(x, 0)\right)^{2}} .
$$

Proof. We will apply maximum principle to the quantity

$$
G=\frac{\left(\kappa_{1}-\kappa_{2}\right)^{2}}{\left(\kappa_{1}+\kappa_{2}\right)^{2}}
$$

Note that $G$ is a homogeneous degree zero function, so (2.8) applies to give the following evolution equation:

$$
\begin{aligned}
\frac{\partial}{\partial t} G=\dot{F}^{i j} & \nabla_{i} \nabla_{j} G+\left(\dot{G}^{i j} \ddot{F}^{k l, m n}-\dot{F}^{i j} \ddot{G}^{k l, m n}\right) \nabla_{i} h_{k l} \nabla_{j} h_{m n} \\
& -\dot{G}^{i j} \dot{F}^{k l} h_{k l} h_{i}^{p} h_{p j}-\dot{G}^{i j} \dot{F}^{k l} g_{i j} h_{k l}+F \dot{G}^{i j} h_{i}^{k} h_{k j}-F \dot{G}^{i j} g_{i j} .
\end{aligned}
$$

Suppose $p$ is a point in $M$ where a new maximum of $G$ is attained at time $t \in[0, T)$. Choose local normal coordinates for $M$ near $p$ such that $h_{i j}(p, t)=\operatorname{diag}\left(\kappa_{1}, \kappa_{2}\right)$. We now estimate the second term on the right-hand side of the above evolution equation. The computation of this term will require results from [7] which describe the components of $F$ and $G$ in the above orthonormal frame:

$$
\begin{aligned}
\ddot{F}^{11,11} & =\frac{\partial^{2} F}{\partial \kappa_{1}^{2}}, \\
\ddot{F}^{11,22}=\ddot{F}^{22,11} & =\frac{\partial^{2} F}{\partial \kappa_{1} \partial \kappa_{2}}, \\
\ddot{F}^{22,22} & =\frac{\partial^{2} F}{\partial \kappa_{2}^{2}}, \\
\ddot{F}^{12,12}=\ddot{F}^{21,21} & =\frac{\frac{\partial F}{\partial \kappa_{1}}-\frac{\partial F}{\partial \kappa_{2}}}{\kappa_{1}-\kappa_{2}} .
\end{aligned}
$$


The last of these identities is to be interpreted as a limit if $\kappa_{1}=\kappa_{2}$. It follows that the second term on the right-hand side of the evolution equation for $G$ are as follows:

$$
\begin{aligned}
& Q=\left(\dot{G}^{i j} \ddot{F}^{k l, m n}-\dot{F}^{i j} \ddot{G}^{k l, m n}\right) \nabla_{i} h_{k l} \nabla_{j} h_{m n} \\
& =\left(\frac{\partial G}{\partial \kappa_{1}} \frac{\partial^{2} F}{\partial \kappa_{1}^{2}}-\frac{\partial F}{\partial \kappa_{1}} \frac{\partial^{2} G}{\partial \kappa_{1}^{2}}\right)\left(\nabla_{1} h_{11}\right)^{2}+\left(\frac{\partial G}{\partial \kappa_{1}} \frac{\partial^{2} F}{\partial \kappa_{2}^{2}}-\frac{\partial F}{\partial \kappa_{1}} \frac{\partial^{2} G}{\partial \kappa_{2}^{2}}\right)\left(\nabla_{1} h_{22}\right)^{2} \\
& +2\left(\frac{\partial G}{\partial \kappa_{1}} \frac{\partial^{2} F}{\partial \kappa_{1} \partial \kappa_{2}}-\frac{\partial F}{\partial \kappa_{1}} \frac{\partial^{2} G}{\partial \kappa_{1} \partial \kappa_{2}}\right) \nabla_{1} h_{11} \nabla_{1} h_{22} \\
& +\left(\frac{\partial G}{\partial \kappa_{2}} \frac{\partial^{2} F}{\partial \kappa_{1}^{2}}-\frac{\partial F}{\partial \kappa_{2}} \frac{\partial^{2} G}{\partial \kappa_{1}^{2}}\right)\left(\nabla_{2} h_{11}\right)^{2}+\left(\frac{\partial G}{\partial \kappa_{2}} \frac{\partial^{2} F}{\partial \kappa_{2}^{2}}-\frac{\partial F}{\partial \kappa_{2}} \frac{\partial^{2} G}{\partial \kappa_{2}^{2}}\right)\left(\nabla_{2} h_{22}\right)^{2} \\
& +2\left(\frac{\partial G}{\partial \kappa_{2}} \frac{\partial^{2} F}{\partial \kappa_{1} \partial \kappa_{2}}-\frac{\partial F}{\partial \kappa_{2}} \frac{\partial^{2} G}{\partial \kappa_{1} \partial \kappa_{2}}\right) \nabla_{2} h_{11} \nabla_{2} h_{22} \\
& +2 \frac{\frac{\partial G}{\partial \kappa_{1}} \frac{\partial F}{\partial \kappa_{2}}-\frac{\partial G}{\partial \kappa_{2}} \frac{\partial F}{\partial \kappa_{1}}}{\kappa_{2}-\kappa_{1}}\left(\nabla_{1} h_{12}\right)^{2}+2 \frac{\frac{\partial G}{\partial \kappa_{1}} \frac{\partial F}{\partial \kappa_{2}}-\frac{\partial G}{\partial \kappa_{2}} \frac{\partial F}{\partial \kappa_{1}}}{\kappa_{2}-\kappa_{1}}\left(\nabla_{2} h_{12}\right)^{2} .
\end{aligned}
$$

Now we note

$$
F=\left(1-\frac{1}{K}\right) \tilde{F}=\tilde{F}-\frac{\tilde{F}}{K}
$$

Let $S=\frac{\tilde{F}}{K}$, so that

$$
\begin{aligned}
Q & =\left(\dot{G}^{i j} \ddot{F}^{k l, m n}-\dot{F}^{i j} \ddot{G}^{k l, m n}\right) \nabla_{i} h_{k l} \nabla_{j} h_{m n} \\
& =\left(\dot{G}^{i j} \ddot{\tilde{F}}^{k l, m n}-\dot{\tilde{F}}^{i j} \ddot{G}^{k l, m n}\right) \nabla_{i} h_{k l} \nabla_{j} h_{m n}-\left(\dot{G}^{i j} \ddot{S}^{k l, m n}-\dot{S}^{i j} \ddot{G}^{k l, m n}\right) \nabla_{i} h_{k l} \nabla_{j} h_{m n} \\
& =Q_{\tilde{F}}-Q_{S}
\end{aligned}
$$

where

$$
Q_{S}=\left(\dot{G}^{i j} \ddot{S}^{k l, m n}-\dot{S}^{i j} \ddot{G}^{k l, m n}\right) \nabla_{i} h_{k l} \nabla_{j} h_{m n} .
$$

At a maximum point ( $p, t)$ of $G, G$ is nonzero (otherwise $M_{t}$ is a sphere and the proof is trivial) and it can be assumed without loss of generality $\kappa_{1}>\kappa_{2}$ because the maximum point is not umbilic. The gradient conditions on $G$ then give two equations:

$$
\nabla_{1} h_{11}=-\frac{\frac{\partial G}{\partial \kappa_{2}}}{\frac{\partial G}{\partial \kappa_{1}}} \nabla_{1} h_{22}, \quad \nabla_{2} h_{22}=-\frac{\frac{\partial G}{\partial \kappa_{1}}}{\frac{\partial G}{\partial \kappa_{2}}} \nabla_{2} h_{11}
$$

The degree zero homogeneity of $G$ implies by the Euler relation that

$$
\kappa_{1} \frac{\partial G}{\partial \kappa_{1}}+\kappa_{2} \frac{\partial G}{\partial \kappa_{2}}=0
$$

Homogeneity also implies the identity

$$
\kappa_{1}^{2} \frac{\partial^{2} G}{\partial \kappa_{1}^{2}}+\kappa_{2}^{2} \frac{\partial^{2} G}{\partial \kappa_{2}^{2}}+2 \kappa_{1} \kappa_{2} \frac{\partial^{2} G}{\partial \kappa_{1} \partial \kappa_{2}}=0 .
$$

Similarly, the degree 1 homogeneity of $\tilde{F}$ and the degree -1 homogeneity of $S$ give the following identities:

$$
\frac{\partial^{2} \tilde{F}}{\partial \kappa_{1}^{2}}=-\frac{\kappa_{2}}{\kappa_{1}} \frac{\partial^{2} \tilde{F}}{\partial \kappa_{1} \partial \kappa_{2}}, \quad \frac{\partial^{2} \tilde{F}}{\partial \kappa_{2}^{2}}=-\frac{\kappa_{1}}{\kappa_{2}} \frac{\partial^{2} \tilde{F}}{\partial \kappa_{1} \partial \kappa_{2}}, \quad \kappa_{1} \frac{\partial \tilde{F}}{\partial \kappa_{1}}+\kappa_{2} \frac{\partial \tilde{F}}{\partial \kappa_{2}}=\tilde{F}
$$


and

$$
\kappa_{1}^{2} \frac{\partial^{2} S}{\partial \kappa_{1}^{2}}+2 \kappa_{1} \kappa_{2} \frac{\partial^{2} S}{\partial \kappa_{1} \kappa_{2}}+\kappa_{2}^{2} \frac{\partial^{2} S}{\partial \kappa_{2}^{2}}=2 S, \quad \kappa_{1} \frac{\partial S}{\partial \kappa_{1}}+\kappa_{2} \frac{\partial S}{\partial \kappa_{2}}=-S .
$$

Substituting these expressions into $Q_{\tilde{F}}$ and $Q_{S}$ and applying the Codazzi symmetries

$$
\nabla_{1} h_{12}=\nabla_{2} h_{11} \quad \text { and } \quad \nabla_{2} h_{12}=\nabla_{1} h_{22}
$$

we obtain

$$
Q_{\tilde{F}}=\frac{2 \tilde{F} \frac{\partial G}{\partial \kappa_{1}}}{\kappa_{2}\left(\kappa_{2}-\kappa_{1}\right)}\left(\nabla_{1} h_{22}\right)^{2}+\frac{2 \tilde{F} \frac{\partial G}{\partial \kappa_{1}}}{\kappa_{2}\left(\kappa_{2}-\kappa_{1}\right)}\left(\nabla_{2} h_{11}\right)^{2}
$$

and

$$
Q_{S}=\frac{\partial G}{\partial \kappa_{1}}\left(\frac{2 S}{\kappa_{2}^{2}}-\frac{2 S}{\kappa_{2}\left(\kappa_{2}-\kappa_{1}\right)}\right)\left(\nabla_{1} h_{22}\right)^{2}+\frac{\partial G}{\partial \kappa_{2}}\left(\frac{2 S}{\kappa_{1}^{2}}-\frac{2 S}{\kappa_{1}\left(\kappa_{2}-\kappa_{1}\right)}\right)\left(\nabla_{2} h_{11}\right)^{2} .
$$
as follows:

The derivatives of the function $G=\frac{\left(\kappa_{1}-\kappa_{2}\right)^{2}}{\left(\kappa_{1}+\kappa_{2}\right)^{2}}$ with respect to $\kappa_{1}$ and $\kappa_{2}$ can be computed

$$
\frac{\partial G}{\partial \kappa_{1}}=\frac{4 \kappa_{2}\left(\kappa_{1}-\kappa_{2}\right)}{\left(\kappa_{1}+\kappa_{2}\right)^{3}}, \quad \frac{\partial G}{\partial \kappa_{2}}=\frac{4 \kappa_{1}\left(\kappa_{2}-\kappa_{1}\right)}{\left(\kappa_{1}+\kappa_{2}\right)^{3}} .
$$

This gives the following:

$$
\begin{aligned}
Q_{\tilde{F}} & =-\frac{8 \tilde{F}}{\left(\kappa_{1}+\kappa_{2}\right)^{3}}\left(\left(\nabla_{1} h_{22}\right)^{2}+\left(\nabla_{2} h_{11}\right)^{2}\right), \\
Q_{S} & =\frac{8 \tilde{F}}{\left(\kappa_{1}+\kappa_{2}\right)^{3} \kappa_{2}^{2}}\left(\nabla_{1} h_{22}\right)^{2}+\frac{8 \tilde{F}}{\left(\kappa_{1}+\kappa_{2}\right)^{3} \kappa_{1}^{2}}\left(\nabla_{2} h_{11}\right)^{2} .
\end{aligned}
$$

In particular, $Q_{\tilde{F}}$ is non-positive and $Q_{S}$ is non-negative, so $Q=Q_{\tilde{F}}-Q_{S} \leq 0$. Now we consider the terms on the second line of (5.3):

$$
\begin{aligned}
Z=-\dot{G}^{i j} \dot{F}^{k l} h_{k l} h_{i}^{p} h_{p j}-\dot{G}^{i j} \dot{F}^{k l} g_{i j} h_{k l}+F \dot{G}^{i j} h_{i}^{k} h_{k j}-F \dot{G}^{i j} g_{i j} \\
=\dot{G}^{i j} h_{i}^{p} h_{p j}\left(-\dot{F}^{k l} h_{k l}+F\right)-\dot{G}^{i j} g_{i j}\left(\dot{F}^{k l} h_{k l}+F\right) \\
=\left(\frac{\partial G}{\partial \kappa_{1}} \kappa_{1}^{2}+\frac{\partial G}{\partial \kappa_{2}} \kappa_{2}^{2}\right)\left(-\frac{\partial F}{\partial \kappa_{1}} \kappa_{1}-\frac{\partial F}{\partial \kappa_{2}} \kappa_{2}+F\right) \\
\quad-\left(\frac{\partial G}{\partial \kappa_{1}}+\frac{\partial G}{\partial \kappa_{2}}\right)\left(\frac{\partial F}{\partial \kappa_{1}} \kappa_{1}+\frac{\partial F}{\partial \kappa_{2}} \kappa_{2}+F\right) .
\end{aligned}
$$

The derivatives of the function $F=\tilde{F}\left(1-\frac{1}{K}\right)$ with respect to $\kappa_{1}$ and $\kappa_{2}$ are as follows:

$$
\frac{\partial F}{\partial \kappa_{1}}=\frac{\partial \tilde{F}}{\partial \kappa_{1}}\left(1-\frac{1}{K}\right)+\frac{\tilde{F} \kappa_{2}}{K^{2}}, \quad \frac{\partial F}{\partial \kappa_{2}}=\frac{\partial \tilde{F}}{\partial \kappa_{2}}\left(1-\frac{1}{K}\right)+\frac{\tilde{F} \kappa_{1}}{K^{2}} .
$$

Substituting the expression (5.4) and (5.5) into the expression for $Z$, we can obtain

$$
Z=-\frac{2 \tilde{F}}{K} \frac{4 \kappa_{1} \kappa_{2}\left(\kappa_{1}-\kappa_{2}\right)^{2}}{\left(\kappa_{1}+\kappa_{2}\right)^{3}}+2 \tilde{F} \frac{4\left(\kappa_{1}-\kappa_{2}\right)^{2}}{\left(\kappa_{1}+\kappa_{2}\right)^{3}}=0 .
$$

Therefore by the maximum principle, the maximum of $G$ is non-increasing. 
Corollary 9. For a smooth compact strictly convex surface $M_{t}$ in $\mathbb{H}^{3}$, flowing according to $\frac{\partial X}{\partial t}=-F v$, there exists a constant $C_{6}=C_{6}\left(M_{0}\right)$ such that

$$
0<\frac{1}{C_{6}} \leq \frac{\kappa_{2}}{\kappa_{1}} \leq C_{6}
$$

Proof. This is a direct consequence of Theorem 8.

5.2. Uniform parabolicity. We next deduce that the evolution equation (5.1) is uniformly parabolic: Since $F=(1-1 / K) \tilde{F}$, we have

$$
\dot{F}^{i j}=\left(1-\frac{1}{K}\right) \dot{\tilde{F}}^{i j}+\frac{\tilde{F}}{K^{2}} \dot{K}^{i j} .
$$

The set

$$
\mathcal{C}=\left\{A:|A|=1, \frac{1}{C_{6}} \leq \frac{\kappa_{2}(A)}{\kappa_{1}(A)} \leq C_{6}\right\}
$$

is compact, so the eigenvalues of $\dot{\tilde{F}}^{i j}$ attain a finite maximum and a positive minimum value on this set. Since $\tilde{F}$ is homogeneous of degree one, we have

$$
\dot{\tilde{F}}^{i j}(h)=\dot{\tilde{F}}^{i j}\left(\frac{h}{|h|}\right) .
$$

Since $h /|h| \in \mathcal{C}$, we have $C_{-} g^{i j} \leq \dot{\tilde{F}}^{i j} \leq C_{+} g^{i j}$ for some $0<C_{-} \leq C_{+}$.

Similarly, by homogeneity and the pinching ratio bound, we have that the eigenvalues of $\frac{\tilde{F}}{K^{2}} \dot{K}^{i j}$ are in an interval of the form $\left[\tilde{C}_{-} / K, \tilde{C}_{+} / K\right]$ for some $0<\tilde{C}_{-} \leq \tilde{C}_{+}$. This gives the bounds

$$
\left[\left(1-\frac{1}{K}\right) C_{-}+\frac{\tilde{C}_{-}}{K}\right] g^{i j} \leq \dot{F}^{i j} \leq\left[\left(1-\frac{1}{K}\right) C_{+}+\frac{\tilde{C}_{+}}{K}\right] g^{i j} .
$$

Since $K>1$, the bracket on the right-hand side is bounded and the bracket on the left is bounded away from zero.

5.3. Speed bounds and positive scalar curvature. The next important result is a lower bound on the speed: From equation (2.6) and the estimates of the previous section, we have

$$
\frac{\partial}{\partial t} F \geq \dot{F}^{i j} \nabla_{i} \nabla_{j} F-C F
$$

for some $C$. Therefore $\inf _{x \in M} F(x, t) \geq \mathrm{e}^{-C t} \inf _{x \in M} F(x, 0)$, and since the maximal time of existence is finite, the speed $F$ has a positive lower bound on the entire interval of existence.

It follows also that $K-1$ has a positive lower bound throughout the evolution, since $(K-1)=\frac{K}{\widetilde{F}} F$. By the pinching ratio bound we have

$$
\frac{K}{\tilde{F}} \geq C \tilde{F} \geq C F
$$

for some $C$, so $K-1 \geq C F^{2}$ has a positive lower bound.

5.4. Convergence. The argument for convergence is now similar to that given for mean curvature flow above: First, the regularity estimates of [6], together with Schauder estimates, 
apply to show that the solution continues to exist as long as the speed remains bounded. We can therefore rescale at a sequences of times approaching the final time on which $F$ attains new spatial maximum values approaching infinity, and product a limit which is a solution of the flow $\frac{\partial X}{\partial t}=-\tilde{F} v$ in Euclidean space, with ratio of principal curvatures bounded. The result of [20] implies that the hypersurfaces of the limiting solution are compact, and the monotonicity of the pinching ratio implies that the limit solution is a shrinking sphere.

\section{Mean curvature flow $(n>2)$}

In this section, we consider the higher-dimensional situation of convex hypersurfaces with positive Ricci curvature in the hyperbolic space $\mathbb{H}^{n+1}$. We restrict our attention to the case of the mean curvature flow $(F=H)$.

6.1. Preserving positive Ricci curvature. We begin by showing that the condition of positive Ricci curvature is preserved under the mean curvature flow. The positive Ricci curvature condition can be written as follows (since the normal direction was chosen to make all principal curvatures positive):

$$
\Omega=\{\operatorname{Ric}>0\}=\bigcap_{i=1}^{n}\left\{\kappa_{i}>0\right\} \cap \bigcap_{i=1}^{n}\left\{H-\kappa_{i}-\frac{n-1}{\kappa_{i}}>0\right\} .
$$

Each of the sets in this intersection is the super-level set of a concave function, and hence is a convex set. Therefore $\Omega$ is a symmetric convex subset of $\mathbb{R}^{n}$. It follows that the set of bilinear forms representing second fundamental forms giving positive Ricci curvature is an $O(n)$-invariant convex subset of $\operatorname{Sym}_{2}\left(\mathbb{R}^{n}\right)$. To apply the vector bundle maximum principle as in [11, Section 7.5.3], it remains only to prove that the vector field $Q$ representing the reaction terms in equation (4.2) points into $\Omega$ at any boundary point. That is, we must show that at a boundary point where one of the defining functions $f_{i}=\kappa_{i}\left(H-\kappa_{i}\right)-(n-1)$ vanishes, the derivative $Q f_{i}$ of $f_{i}$ in direction $Q$ is non-negative. The $k$ th component of $Q$ at the point $\left(\kappa_{1}, \ldots, \kappa_{n}\right)$ is

$$
Q\left(\kappa_{k}\right)=\kappa_{k}\left(|A|^{2}+n\right)-2 H .
$$

Therefore we have

$$
\begin{aligned}
& Q f_{i}=\left(\kappa_{i}\left(|A|^{2}+n\right)-2 H\right)\left(H-\kappa_{i}\right)+\kappa_{i} \sum_{j \neq i}\left(\kappa_{j}\left(|A|^{2}+n\right)-2 H\right) \\
&= 2 \kappa_{i}\left(H-\kappa_{i}\right)\left(|A|^{2}+n\right)-2 H\left(H-\kappa_{i}\right)-2(n-1) \kappa_{i} H \\
&= 2\left(\kappa_{i}\left(H-\kappa_{i}\right)-(n-1)\right)|A|^{2}+2(n-1)|A|^{2}+2 n \kappa_{i}\left(H-\kappa_{i}\right) \\
& \quad-2\left(H-\kappa_{i}\right)^{2}-2 \kappa_{i}\left(H-\kappa_{i}\right)-2(n-1) \kappa_{i}\left(H-\kappa_{i}\right)-2(n-1) \kappa_{i}^{2} \\
&= 2\left(\kappa_{i}\left(H-\kappa_{i}\right)-(n-1)\right)|A|^{2}+2(n-1) \sum_{j \neq i} \kappa_{j}^{2}-\left(\sum_{j \neq i} \kappa_{j}\right)^{2} \\
&= 2 f_{i}|A|^{2}+\sum_{j, k \neq i}\left(\kappa_{j}-\kappa_{k}\right)^{2} \\
& \geq 2 f_{i}|A|^{2} .
\end{aligned}
$$


In particular, since $f_{i}=0$, we have $Q f_{i} \geq 0$ and the maximum principle therefore applies to prove that the principal curvature remain in $\Omega$, and the condition of positive Ricci curvature is preserved under the mean curvature flow.

6.2. The pinching estimate. The crucial step in controlling the hypersurfaces is to obtain an estimate which bounds the ratio of principal curvatures under the mean curvature flow, for any initial hypersurface with positive Ricci curvature. The precise result is as follows:

Proposition 4. For any compact solution of the mean curvature flow with positive Ricci curvature, there exists an $\varepsilon>0$ such that the following inequality holds at every point $(x, t) \in M \times[0, T):$

$$
\kappa_{k}\left(H-\kappa_{k}\right)-(n-1) \geq \varepsilon H\left(\kappa_{i}-\kappa_{j}\right)
$$

for all $i, j$ and $k$.

Proof. We will again employ the vector bundle maximum principle, noting that by compactness and the positivity of the Ricci curvature the inequality holds for sufficiently small $\varepsilon>0$ when $t=0$. We must first show that the inequality defines a convex set. Dividing inequality (6.3) by $H$, we find that the set we are trying to preserve can be written in the form

$$
\Omega_{\varepsilon}=\bigcap_{i}\left\{\kappa_{i}>0\right\} \cap \bigcap_{i, j, k}\left\{\left(\kappa_{k}-\frac{\kappa_{k}^{2}}{H}-\frac{n-1}{H}\right)-\varepsilon \kappa_{i}+\varepsilon \kappa_{j} \geq 0\right\} .
$$

The function in the last bracket is concave: The terms $\kappa_{k}-\varepsilon \kappa_{j}+\varepsilon \kappa_{i}$ are linear, and $-1 / H$ is clearly concave. The function $-\kappa_{k}^{2} / H$ is also concave, since it is homogeneous of degree one and manifestly concave on the hyperplane $\{H=1\}$. Therefore $\Omega_{\varepsilon}$ is an intersection of superlevel sets of concave functions, hence is a convex set.

It remains only to prove that the vector field $Q$ points into $\Omega_{\varepsilon}$ at boundary points. Equivalently, we must show that at a boundary point $\left(\kappa_{1}, \ldots, \kappa_{n}\right)$ where one of the defining functions $f_{i, j, k}=\kappa_{k}\left(H-\kappa_{k}\right)-(n-1)-\varepsilon H\left(\kappa_{i}-\kappa_{j}\right)$ is zero, the derivative $Q f_{i, j, k}$ is non-negative where $Q$ is given by (6.1). We note that $f_{i, j, k}=f_{k}-\varepsilon \varphi_{i, j}$ where $f_{k}$ is defined as in Section 6.1 and $\varphi_{i, j}=H\left(\kappa_{i}-\kappa_{j}\right)$. Since $Q \kappa_{i}=\kappa_{i}\left(|A|^{2}+n\right)-2 H$, we have

$$
Q H=H\left(|A|^{2}+n\right)-2 n H=H\left(|A|^{2}-n\right) .
$$

Also we have

$$
Q\left(\kappa_{i}-\kappa_{j}\right)=\kappa_{i}\left(|A|^{2}+n\right)-2 H-\kappa_{j}\left(|A|^{2}+n\right)+2 H=\left(\kappa_{i}-\kappa_{j}\right)\left(|A|^{2}+n\right) .
$$

Combining these gives

$$
\begin{aligned}
Q \varphi_{i, j} & =(Q H)\left(\kappa_{i}-\kappa_{j}\right)+H Q\left(\kappa_{i}-\kappa_{j}\right) \\
& =H\left(\kappa_{i}-\kappa_{j}\right)\left(|A|^{2}-n\right)+H\left(\kappa_{i}-\kappa_{j}\right)\left(|A|^{2}+n\right) \\
& =2 H\left(\kappa_{i}-\kappa_{j}\right)|A|^{2} \\
& =2 g_{i, j}|A|^{2} .
\end{aligned}
$$

Combining this with equation 6.2 gives

$$
Q f_{i, j, k}=Q f_{k}-\varepsilon Q g_{i, j} \geq 2 f_{k}|A|^{2}-2 \varepsilon g_{i, j}|A|^{2}=2 f_{i, j, k}|A|^{2}=0 .
$$

The maximum principle therefore applies, and we have proved (6.3). 
Corollary 10. Under the assumptions of Proposition 4 there exists a constant $C>0$ such that

$$
\frac{1}{C} \leq \frac{\kappa_{i}(x, t)}{\kappa_{j}(x, t)} \leq C
$$

for all $x \in M$ and $t \in[0, T)$.

Proof. Labelling the principal curvatures in increasing order, we have

$$
H\left(\kappa_{n}-\kappa_{1}\right) \leq \frac{1}{\varepsilon}\left(\kappa_{1}\left(H-\kappa_{1}\right)-(n-1)\right) \leq \frac{1}{\varepsilon} \kappa_{1}\left(H-\kappa_{1}\right) \leq \frac{1}{\varepsilon} \kappa_{1} H .
$$

Dividing through by $H$ we find

$$
\kappa_{n}-\kappa_{1} \leq \frac{1}{\varepsilon} \kappa_{1}
$$

and therefore

$$
\kappa_{n} \leq\left(1+\frac{1}{\varepsilon}\right) \kappa_{1},
$$

so the corollary holds with $C=1+\frac{1}{\varepsilon}$.

6.3. Rescaling and convergence. The argument for convergence given in Section 4.2 applies for this case without change.

\section{References}

[1] R. Alessandroni and C. Sinestrari, Evolution of hypersurfaces by powers of the scalar curvature, Ann. Sc. Norm. Super. Pisa Cl. Sci. (5) 9 (2010), no. 3, 541-571.

[2] B. Andrews, Contraction of convex hypersurfaces in Euclidean space, Calc. Var. Partial Differential Equations 2 (1994), no. 2, 151-171.

[3] B. Andrews, Contraction of convex hypersurfaces in Riemannian spaces, J. Differential Geom. 39 (1994), no. 2, 407-431.

[4] B. Andrews, Gauss curvature flow: The fate of the rolling stones, Invent. Math. 138 (1999), no. 1, 151-161.

[5] B. Andrews, Positively curved surfaces in the three-sphere, in: Proceedings of the international congress of mathematicians, ICM 2002. Vol. II: Invited lectures (Beijing 2002), Higher Education Press, Beijing (2002), 221-230.

[6] B. Andrews, Fully nonlinear parabolic equations in two space variables, preprint 2004, http: //arxiv .org/ abs/math/0402235.

[7] B. Andrews, Pinching estimates and motion of hypersurfaces by curvature functions, J. reine angew. Math. 608 (2007), 17-33.

[8] B. Andrews, Moving surfaces by non-concave curvature functions, Calc. Var. Partial Differential Equations 39 (2010), no. 3-4, 649-657.

[9] B. Andrews and C. Baker, Mean curvature flow of pinched submanifolds to spheres, J. Differential Geom. 85 (2010), no. 3, 357-395.

[10] B. Andrews and X. Chen, Surfaces moving by powers of Gauss curvature, Pure Appl. Math. Q. 8 (2012), no. 4, 825-834.

[11] B. Andrews and C. Hopper, The Ricci flow in Riemannian geometry, Lecture Notes in Math. 2011, Springer, Heidelberg 2011.

[12] B. Andrews and J. McCoy, Convex hypersurfaces with pinched principal curvatures and flow of convex hypersurfaces by high powers of curvature, Trans. Amer. Math. Soc. 364 (2012), no. 7, 3427-3447.

[13] C. Baker, The mean curvature flow of submanifolds of high codimension, Ph.D. thesis, The Australian National University, 2010, http://arxiv .org/abs/1104.4409.

[14] E. Cabezas-Rivas and V. Miquel, Volume preserving mean curvature flow in hyperbolic space, preprint 2006, http://arxiv.org/abs/math/0611216. 
[15] B. Chow, Deforming convex hypersurfaces by the $n$th root of the Gaussian curvature, J. Differential Geom. 22 (1985), no. 1, 117-138.

[16] B. Chow, Deforming convex hypersurfaces by the square root of the scalar curvature, Invent. Math. 87 (1987), no. 1, 63-82.

[17] E. DiBenedetto and A. Friedman, Hölder estimates for nonlinear degenerate parabolic systems, J. reine angew. Math. 357 (1985), 1-22.

[18] K. Ecker and G. Huisken, Interior estimates for hypersurfaces moving by mean curvature, Invent. Math. 105 (1991), no. 3, 547-569.

[19] R. S. Hamilton, Four-manifolds with positive curvature operator, J. Differential Geom. 24 (1986), no. 2, 153-179.

[20] R. S. Hamilton, Convex hypersurfaces with pinched second fundamental form, Commun. Anal. Geom. 2, no. 1, 167-172 (1994).

[21] G. Huisken, Flow by mean curvature of convex surfaces into spheres, J. Differential Geom. 20 (1984), no. 1, 237-266.

[22] G. Huisken, Contracting convex hypersurfaces in Riemannian manifolds by their mean curvature, Invent. Math. 84 (1986), no. 3, 463-480.

[23] G. Huisken, Deforming hypersurfaces of the sphere by their mean curvature, Math. Z. 195 (1987), 205-219.

[24] G. Huisken and A. Polden, Geometric evolution equations for hypersurfaces, in: Calculus of variations and geometric evolution problems. Lectures given at the 2nd session of the Centro Internazionale Matematico Estivo (CIME) (Cetraro 1996), Lecture Notes Math. 1713, Springer, Berlin (1999), 45-84.

[25] Q.-R. Li, Surfaces expanding by the power of the Gauss curvature flow, Proc. Amer. Math. Soc. 138 (2010), no. 11, 4089-4102.

[26] O. C. Schnürer, Surfaces contracting with speed $|A|^{2}$, J. Differential Geom. 71 (2005), no. 3, 347-363.

[27] O.C. Schnürer, Surfaces expanding by the inverse Gauß curvature flow, J. reine angew. Math. 600 (2006), 117-134.

[28] F. Schulze, Convexity estimates for flows by powers of the mean curvature, Ann. Sc. Norm. Super. Pisa Cl. Sci. (5) 5 (2006), no. 2, 261-277.

[29] K. Tso, Deforming a hypersurface by its Gauss-Kronecker curvature, Comm. Pure Appl. Math. 38 (1985), no. 6, 867-882.

[30] C. Wu, D. Tian and G. Li, Forced flow by powers of the $m$ th mean curvature, Armen. J. Math. 3 (2010), no. 2, 61-91.

Ben Andrews, Mathematical Sciences Center, Tsinghua University, Beijing, P. R. China; and Mathematical Sciences Institute, Australia National University, Canberra, Australia e-mail: ben.andrews@anu.edu.au

Xuzhong Chen, Department of Mathematics, East China Normal University, Shanghai, P. R. China e-mail: xuzhong_chen@163.com

Eingegangen 24. Januar 2013, in revidierter Fassung 29. Juli 2014 